\title{
Desarrollo de una Herramienta Informática para la Simulación de Reportes PVT en los Pozos del Oriente Ecuatoriano de la Arena U Inferior
}

\author{
Arteaga, Jimmy ${ }^{1}$ (D) ; Gómez, Franklin ${ }^{1}$ (D); Iza, Anabel ${ }^{1}$ * * iD ; Izurieta, Álvaro ${ }^{2}$ iD ; Jiménez, Tamia ${ }^{1}$ (iD); \\ Valencia, Raúl ${ }^{1}$ iD ; Zurita, Kevin ${ }^{1}$ iD

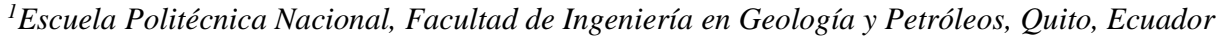 \\ ${ }^{2}$ Halliburton, Quito, Ecuador
}

\begin{abstract}
Resumen: En el presente artículo se describe el desarrollo de un programa que permita la estimación de propiedades de Presión, Volumen y Temperatura (PVT) en los pozos del Oriente Ecuatoriano. El prototipo cuenta con la recopilación de correlaciones más adecuadas para crudo Ecuatoriano, visualización y generación de reportes de los pozos que se encuentren perforados sujeta a información geográfica. Como primer punto, es necesario contar con expresiones matemáticas PVT calibradas a datos de laboratorio correspondientes a la arena U inferior del crudo de la región. Para esta parte, la metodología desarrollada constituye la adquisición, procesamiento y ajuste de datos de información de laboratorio, con el fin de obtener un manejo adecuado de los mismos. La aplicación desarrollada proporcionará la estimación de las propiedades PVT del petróleo más acordes a la Cuenca Oriente. En tal virtud, los resultados experimentales podrán ser utilizados en los diferentes cálculos requeridos para la producción de hidrocarburos de la industria local y para la academia permitirá ampliar las fronteras de estudio.
\end{abstract}

Palabras clave: PVT, propiedades, petróleo, prototipo, correlaciones, metodología.

\section{Development of a Computer Tool for PVT Report Simulation in the Ecuadorian Wells for the Lower U Sand}

\begin{abstract}
This article describes the development of a software that estimates the Pressure, Volume and Temperature (PVT) properties for the Lower U sandstone in Ecuador. The prototype has a collection of PVT correlations more suitable for Ecuadorian Crude Oils, visualization and report generation of the wells based on geographic information. As a first step, it is necessary to have mathematical PVT expressions calibrated to laboratory data. The software developed will provide an estimation of PVT properties to better suit to the Oriente Basin. Therefore, the experimental results can be used in different calculations required for hydrocarbon production of the local industry and for the academy it will allow to expand the study frontiers.
\end{abstract}

Keywords: PVT, properties, oil, software, correlations, methodology.

\section{INTRODUCCIÓN}

Las correlaciones PVT que permiten estimar las propiedades físicas del petróleo son de uso común en la industria como en la academia. La aplicación de estas expresiones matemáticas implica una ventaja y a la par una limitante debido a que los valores estimados pueden mostrar desviaciones significativas respecto del comportamiento real de la propiedad estudiada. Esto se da por que la gran mayoría fueron desarrolladas para crudos de diferentes regiones (i.e. composición química diferente a la del crudo ecuatoriano) lo que limita los rangos de aplicación de cada correlación (Farías \& Merola, 2014).
De la gran variedad de correlaciones disponibles en la literatura ninguna de ellas se ajusta al comportamiento de crudo ecuatoriano, solo proporcionan valores puntuales.

En la presente investigación se modifican los coeficientes originales de las correlaciones de la literatura; las propiedades a evaluar son: relación gas-petróleo $\left(\mathrm{R}_{\mathrm{s}}\right)$, factor volumétrico del petróleo $\left(\mathrm{B}_{\mathrm{o}}\right)$ y compresibilidad del petróleo $\left(\mathrm{C}_{\mathrm{o}}\right)$. Los rangos de presión que se contemplan en el análisis de estas propiedades son mayores, menores e iguales a la presión de burbuja. 
Adicionalmente, una potencial herramienta informática se diseña y desarrolla para recopilar las correlaciones modificadas y toda la información experimental proveniente de los reportes PVT. De la misma forma permitirá realizar la estimación de las propiedades PVT del petróleo ecuatoriano de la arena U inferior. Esta aplicación se limita a realizar estudios PVT en los campos que actualmente se encuentran en desarrollo.

\section{OBJETIVOS}

- Generar un conjunto de correlaciones PVT modificadas al comportamiento del crudo ecuatoriano de la arena $\mathrm{U}$ inferior.

- Generar una base de datos PVT comprensiva.

- Modificar las correlaciones seleccionadas mediante recursos estadísticos y/o numéricos.

- Realizar análisis de datos con el fin de mejorar las correlaciones modificadas en forma general.

- Desarrollar un programa de cómputo para el cálculo de propiedades PVT del petróleo.

\section{METODOLOGÍA}

\subsection{Información de laboratorio (Reportes PVT)}

Los reportes PVT proceden del resultado de pruebas experimentales realizadas a la muestra de fluido (petróleo) considerando tres parámetros de variación presión, volumen y temperatura los mismos que gobiernan el comportamiento volumétrico del fluido desde el reservorio hasta las instalaciones de superficie (Bujowicz, 1988). Las pruebas experimentales que permiten caracterizar el fluido del reservorio (crudo ecuatoriano) son: expansión a composición constante, liberación diferencial, prueba del separador y viscosidad. De cada una de ellas se obtiene información en sus propiedades: presión de burbuja, compresibilidad isotérmica, factor volumétrico y relación de solubilidad y viscosidad en función de la presión (Iza, 2017).

Para el desarrollo de esta investigación se considera el siguiente flujo de trabajo (Iza, 2017):

Recopilación de reportes PVT correspondientes a las muestras de fluido de los diferentes campos petroleros de la Cuenca Oriente. La información obtenida pasa por un proceso de clasificación de muestras, debido a que las muestras de fluido proceden de diferentes fuentes como fondo del pozo, cabezal de producción o separador de producción. Por otro lado, es importante mencionar que las pruebas de laboratorio utilizadas en este estudio corresponden a muestras de fondo que cumplen con las características necesarias para su uso (comportamiento físico real y ensayos de laboratorio completos para el cálculo de las propiedades PVT). Con estos antecedentes varios reportes fueron descartados y un total de 311 son utilizados para posteriores pasos.

\subsection{Validación de información}

Este apartado hace referencia al procesamiento y ajuste de la información, la cual consta de lo siguiente (Izurieta \& Iza, 2017):
- Verificación del comportamiento de curvas de las propiedades físicas del petróleo $\left(\mathrm{B}_{\mathrm{o}}, \mathrm{C}_{\mathrm{o}}, \mathrm{R}_{\mathrm{s}}\right.$, ) con la presión.

- Información completa de pruebas de laboratorio (si se tiene todos los parámetros $\mathrm{B}_{\mathrm{o}}, \mathrm{C}_{\mathrm{o}}, \mathrm{R}_{\mathrm{s}}, \mathrm{U}_{\mathrm{o}}$ ).

- Ecuaciones de ajuste de $\mathrm{B}_{\circ}$ y $\mathrm{R}_{\mathrm{s}}$ de la prueba de liberación diferencial a condiciones de separador.

Las Figuras 1-3 muestran el comportamiento establecido para cada propiedad en análisis en función de la presión.

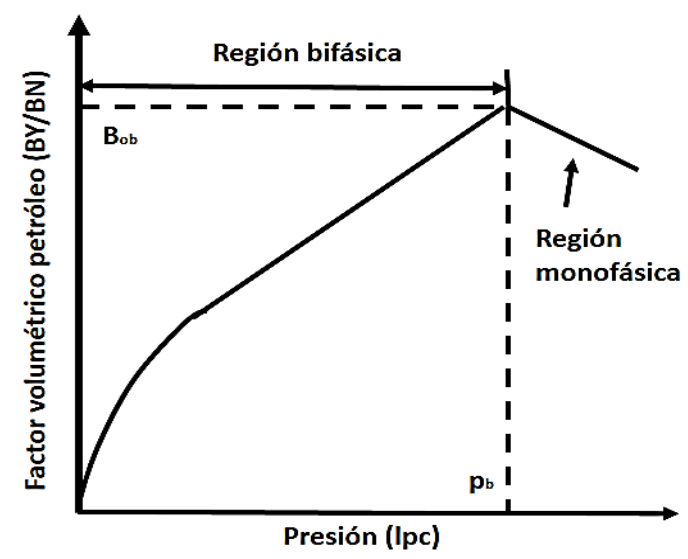

Figura 1. Comportamiento Bo vs P.

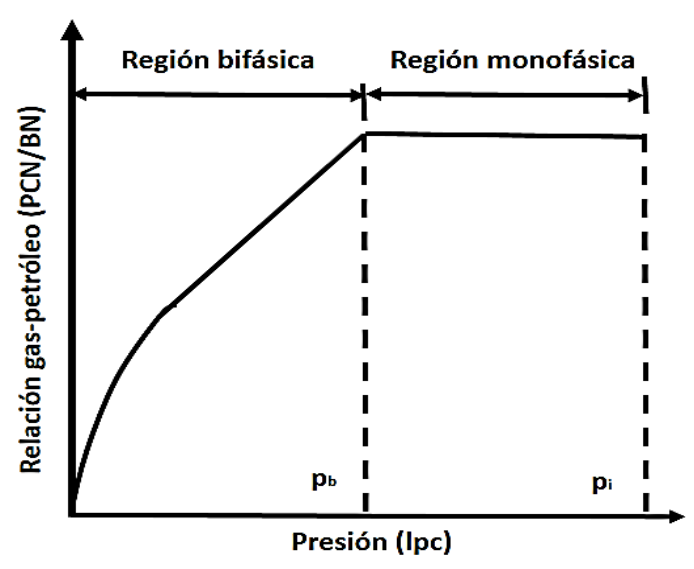

Figura 2. Comportamiento Rs vs P.

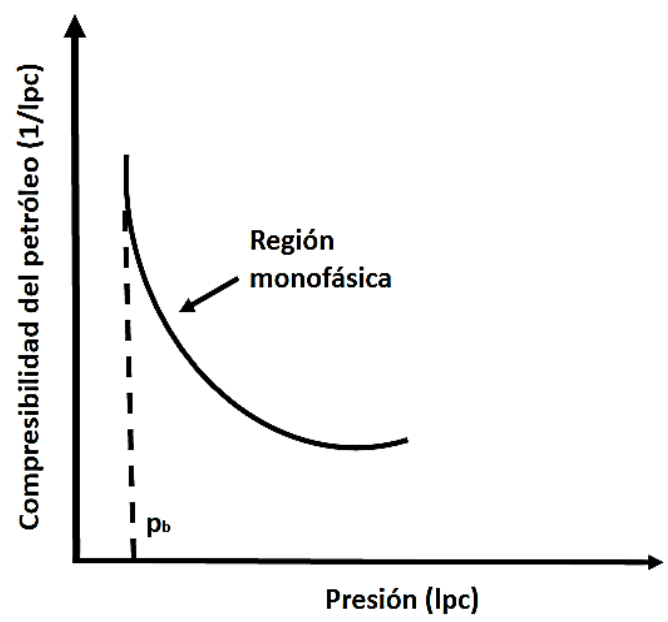

Figura 3. Comportamiento Co vs $\mathrm{P}$. 


\subsection{Correlaciones PVT}

Las expresiones de correlación fueron seleccionadas considerando los parámetros del reservorio como temperatura (T), gravedad específica del petróleo $\left({ }_{-}^{\circ} \mathrm{API}\right)$, gravedad específica del gas $\left(\gamma_{\mathrm{g}}\right)$ y relación de solubilidad $\left(\mathrm{R}_{\mathrm{s}}\right)$ (Izurieta e Iza, 2017), de esta manera se excluye las formulaciones complejas que presenten procedimientos iterativos.

Las correlaciones seleccionadas fueron elegidas considerando lo siguiente y se enlistan en la Tabla 1:

- Para el factor volumétrico del petróleo, modelo de Al Marhoun (1988) y para la relación gas-petróleo, modelo de Standing (1947) respectivamente, se recomiendan en la investigación de Jiménez \& Zurita por tener menor error estadístico en comparación con otros modelos.

Para estas dos propiedades se consideró rangos de presión mayores, menores e iguales a la presión de burbuja.

- Para la compresibilidad de petróleo se trabajó con rangos de presión mayores e iguales a la presión de burbuja, no se contó con información experimental bajo este rango. Kartoatmodjo-Schmidt (1991) es el modelo con menor error estadístico propuesto (Jiménez \& Zurita, 2019).

\begin{tabular}{cc}
\multicolumn{2}{c}{ Tabla 1. Correlaciones PVT del petróleo. } \\
\hline Propiedad & Autor \\
\hline $\begin{array}{c}\text { Relación gas- } \\
\text { petroleo } \mathrm{R}_{\mathrm{s}}\end{array}$ & Petrosky-Farshad 1993 \\
Factor & \\
volumétrico del & Al Marhoun 1988 \\
petróleo $\mathrm{B}_{\mathrm{o}}$ & \\
Compresibilidad & Kartoatmodjo-Schmidt \\
del petróleo $\mathrm{C}_{\mathrm{o}}$ & 1991 \\
\hline
\end{tabular}

\subsection{Modificación de correlaciones PVT}

Las correlaciones seleccionadas por propiedad fueron evaluadas sin modificación y luego con modificación de sus coeficientes originales, para este último se empleó la herramienta Solver-Excel con el fin de calibrar los coeficientes a datos de laboratorio. Para este fin fue necesario realizar una macro que permita la comunicación de la herramienta Solver a cada muestra mediante la aplicación Visual Basic Application (VBA); esto permite determinar el porcentaje de error y si el mismo excede el $10 \%$ se usa la herramienta Solver hasta lograr reducir el error al rango predefinido.

Para las correlaciones modificadas por propiedad se constató los siguientes criterios (Iza, 2017):

- Gráfico valores estimados y valores experimentales y comportamiento de cada propiedad estimada con la presión.

- Porcentaje de error.

- Coeficiente de correlación (r).

\subsection{Desarrollo y descripción del programa}

Previo al desarrollo del prototipo se elaboró una base de datos en el gestor Microsoft Access, el cual cuenta con una estructura lógica y es la encargada de almacenar, administrar y ordenar la información de acuerdo a lo siguiente:

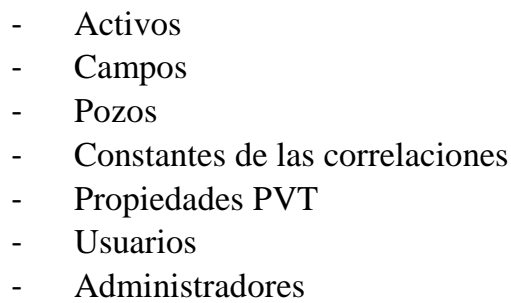

El programa cuenta con 4 etapas importantes:

1. Conexión a la base de datos.

2. Envío de información (Activo, Campo, Pozo, Propiedad) a través del software a la base de datos por medio de procedimientos almacenados (lenguaje de programación).

3. Lectura de la información desde la base de datos hacia la aplicación.

4. Visualización de los resultados (tablas y gráficos).

La aplicación permite la consulta de propiedades con base en los reportes existentes o de requerirse escogerá mediante coordenadas geográficas el pozo A (base de datos) más cercano al pozo B (que no cuenta con datos PVT) asociado a él un grupo de correlaciones para la estimación de las propiedades de petróleo (datos PVT del pozo B).

\section{RESULTADOS Y DISCUSIÓN}

\subsection{Correlaciones PVT modificadas}

Las correlaciones modificadas no cambiaron en forma, los coeficientes fueron adecuados a la información experimental (PVT); es decir para cada muestra se obtuvo un grupo de coeficientes asociados a cada propiedad. Las "Ecuaciones (1$3)$ ) están incluidas en el funcionamiento del programa.

\section{- Factor volumétrico}

Al Marhoun 1988

$$
B o=a+b T+c\left(R s^{d} \gamma_{g}{ }^{e} \gamma_{o}{ }^{f}\right)+g\left(R s^{d} \gamma_{g}{ }^{e} \gamma_{o}{ }^{f}\right)^{h}
$$

\section{- Relación gas-petróleo}

Standing 1947

$$
R s=\left[\gamma_{g}{ }^{a}\left(\frac{P}{b}+c\right) 10^{\left(d A P I^{e}-f T^{g}\right)}\right]^{h}
$$

\section{- Compresibilidad}

Kartoatmodjo - Schmidt 1991

$$
C o=a \times R s^{b} \times P \times T^{c} \times \gamma_{g}{ }^{d}
$$




\subsection{Desarrollo de la aplicación}

La Figura 4 muestra la interfaz del prototipo el cual contiene la base de datos descrita en el punto 3.5 de este trabajo.

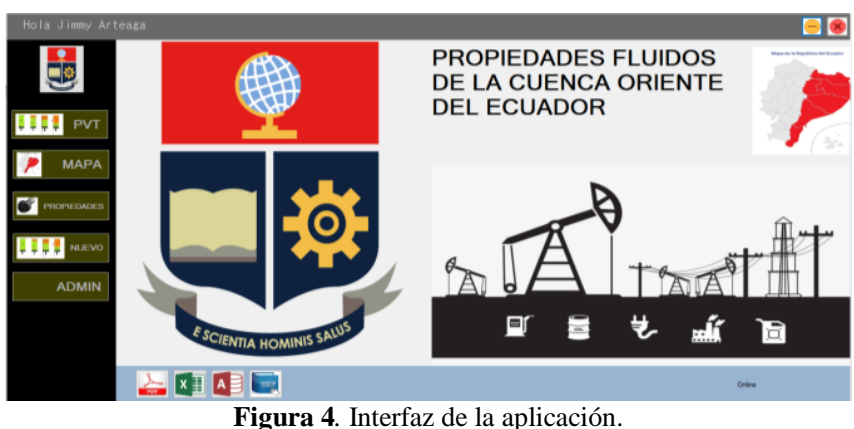

Una de las secciones diseñadas en esta versión del programa es el módulo PVT. En esta sección se observan los resultados como tablas, gráficos, etc., como se muestra en las Figuras 57.

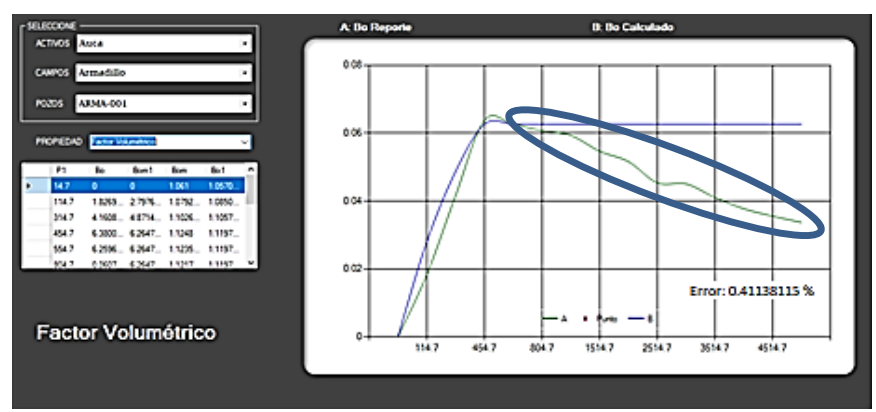

Figura 5. Resultado del Factor volumétrico del petróleo vs Presión.

Pese a que el error presentado para el factor volumétrico es menor al $1 \%$, se evidencia que el comportamiento de esta propiedad para rangos mayores a la presión de burbuja no se ajusta a los datos experimentales por lo que buscándose, disminuye el error aplicando un multiplicador de ajuste para que coincida con los parámetros PVT. De la misma forma que se realizó en un trabajo del 2014 publicado por Austin \& Sunday.

En la Figura 6, se observa el resultado desplegado para la relación gas - petróleo con un error despreciable menor al $1 \%$, al presentarse esto se evidencia la precisión del modelo matemático.

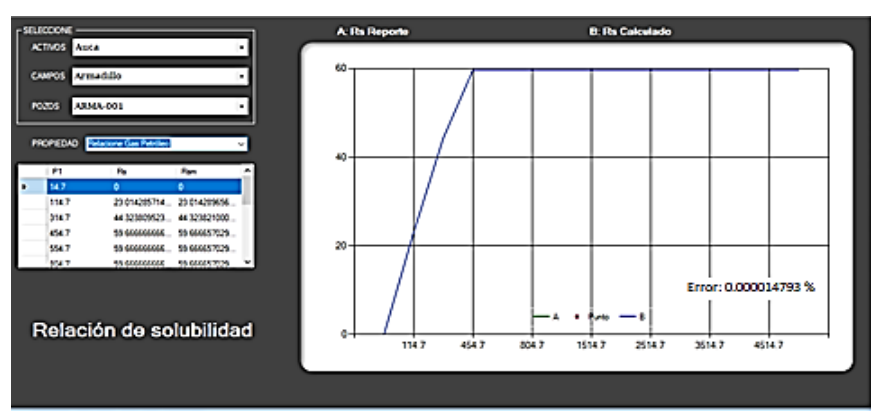

Figura 6. Resultado de la Relación de solubilidad del petróleo vs Presión.

Para la compresibilidad del petróleo se observa que los valores estimados poseen sobrestimación (a leve escala), curva azul sobrepasa la curva verde. El error presentado es aproximadamente del $3 \%$ (Ver Figura 7).

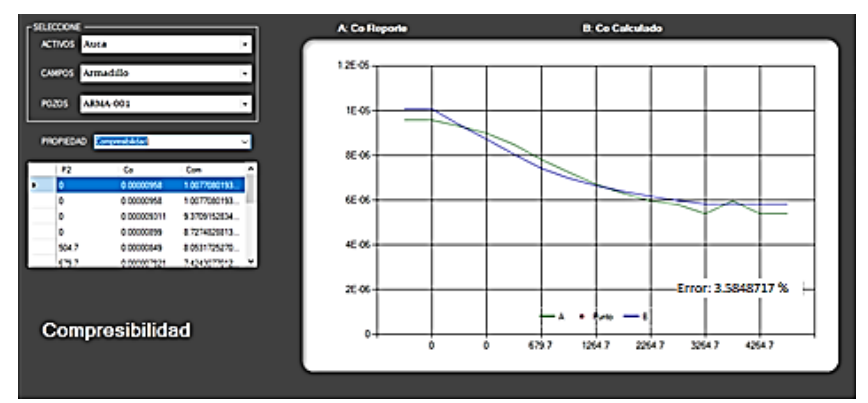

Figura 7. Resultado de la Compresibilidad de petróleo vs Presión.

Las Figura 8 muestra la interfaz de la base de datos de coordenadas geográficas diseñada con Visual Studio, la cual permite seleccionar los reportes existentes o ingresar las coordenadas del pozo en estudio Latitud y Longitud para el cálculo de las propiedades.

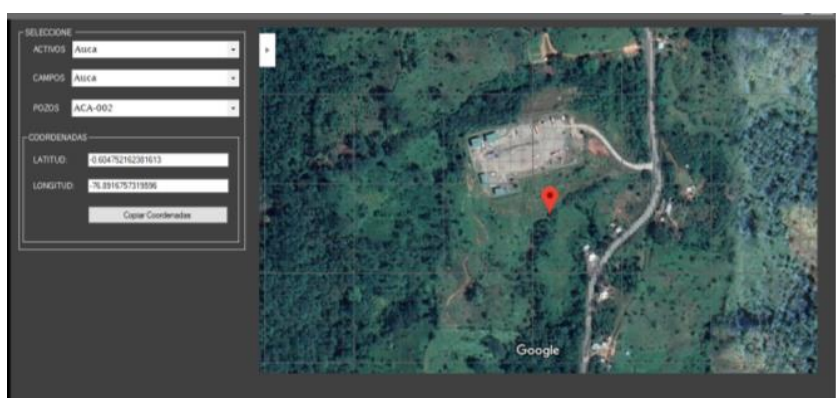

Figura 8. Coordenadas geográficas de los pozos.

La Figura 9 muestra los resultados de un pozo (nuevo o sin PVT) utilizando la base de coordenadas geográficas.

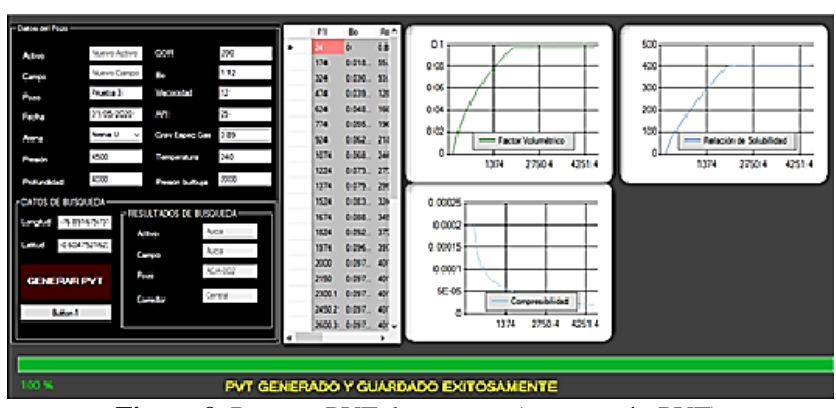

Figura 9. Reporte PVT de un pozo (nuevo o sin PVT).

En el Ecuador existen varios trabajos asociados al análisis, generación y estandarización de correlaciones PVT por campo, arena y área geográfica (como muestra véase, Murillo, J y Yépez, C (2013); Izurieta, A e Iza, A (2017); Iza, A (2017); Jiménez, T y Zurita, K (2019)), que incluyen varios criterios de investigación y herramientas de desarrollo innovadores. La diferencia de este trabajo en comparación con los mencionados anteriormente radica en el despliegue de resultados obtenidos, gráficas, tablas, reportes nuevos, así como la generación de una base de datos comprensiva.

La implementación de nuevos mecanismos como redes neuronales artificiales puede contribuir en la modificación de correlaciones tanto en el aspecto cuantitativo (error) como en el cualitativo (gráfica), recientes publicaciones han ganado popularidad en la Industria Petrolera (Muzammil y Abdulazeez, 2015). 


\section{CONCLUSIONES}

Las correlaciones modificadas fueron desarrolladas para rangos de presión mayores, menores e iguales a la presión de burbuja a excepción de la compresibilidad de petróleo.

La mayoría de correlaciones contemplan parámetros de producción como temperatura, gravedad API, gravedad especifica del gas y presión los mismos que se presentan en los reportes PVT.

Los criterios de evaluación utilizados no solo en este estudio sino en otros trabajos (Izurieta e Iza, 2017; Iza, 2017; Jiménez y Zurita, 2019) presentan gran aceptación en cuanto a la precisión y exactitud de datos y expresiones matemáticas.

El desarrollo del prototipo marca una gran expectativa para la Academia pues será capaz de interactuar de una forma más real e ingenieril.

El cálculo de propiedades PVT es de gran importancia para las diferentes fases de producción de hidrocarburo, el uso de esta aplicación disminuye significativamente la incertidumbre en los mismos.

No siempre se tienen reportes PVT disponibles para todos los reservorios, por ello el software contribuye como una fuente de consulta y cálculo para diferentes proyectos en la Industria Petrolera.

Es importante realizar un análisis de los resultados experimentales (reportes PVT) debido a que no siempre contienen datos confiables debido a las condiciones de muestreo.

Esta es la primera versión del software por lo que es posible expandir el rango de estudio a otros reservorios y mayor cantidad de propiedades.

\section{REFERENCIAS}

Al-Marhoun, M. A. (1988). PVT Correlations for Middle East Crude Oils. J Pet Technol, 40(5), 650-666. https://doi.org/10.2118/13718-PA.

Al-Marhoun, M. A. (2001). Adjustment of Differential Liberation Data to Separator Conditions. Society of Petroleum Engineers, 6(3), 142-146. https://doi.org/10.2118/68234-MS.

Al-Marhoun, M. A. (2003). Evaluation of empirically derived PVT properties for Middle East crude oils. J Pet Sci \& Eng, 42(2004),

209-221. https://doi.org/10.1016/j.petrol.2003.12.012.

Al-Shammasi, A. A. (1999). Bubble Point Pressure and Oil Formation Volume Factor Correlations. Society of Petroleum Engineers, 1-17. https://doi.org/10.2118/53185-MS.

Almehaideb, R. A. (1997). Improved PVT Correlations for UAE Crude Oils. Society of Petroleum Engineers, 109 120. https://doi.org/10.2118/37691-MS.
Austin, K., \& Sunday, I. (2014). Globalization of Black Oil PVT Correlations. Society of Petroleum Engineers, 118. https://doi.org/10.2118/172494-MS.

Beal, C. (1946). The Viscosity of Air, Water, Natural Gas, Crude Oil and Its Associated Gases at Oil Field Temperatures and Pressures. Trans AIME, 165(1), 94115. https://doi.org/10.2118/946094-G.

Beggs, H. D., \& Robinson, J. R. (1975). Estimating the Viscosity of Crude Oil Systems. J Pet Technol, 27(9), 1140-1141. https://doi.org/10.2118/5434-PA.

Bujowicz, R. (1988). PVT Data Generation Reporting and General Uses. Petroleum Society of Canada, 1-14. https://doi.org/10.2118/88-39-66.

Dindoruk, B., \& Christman, P. G. (2001). PVT Properties and Viscosity Correlations for Gulf of Mexico Oils. Society of Petroleum Engineer $s, \quad 1-14$. https://doi.org/10.2118/71633-MS.

Dokla, M. E., \& Osman, M. E. (1992). Correlation of PVT Properties for UAE Crudes (includes associated papers 26135 and 26316). Society of Petroleum Engineers, 7(1), 41-46. https://doi.org/10.2118/20989-PA.

Farías, L., \& Merola, V. (2014). Propiedades de los fluidos del Yacimiento, 212-248; 253-254. Barcelona: Reverté S.A.

Frashad, F., Le Blanc, J. L., Garber, J. D., \& Osorio, J. G. (1996). Empirical PVT Correlations for Colombian Crude Oils. Society of Petroleum Engineers, 311-320. https://doi.org/10.2118/36105-MS.

Ghetto, G. De, Paone, F., Villa, M., \& S.p.A., A. (1995). Pressure-Volume-Temperature Correlations for Heavy and Extra Heavy Oils. Society of Petroleum Engineers. https://doi.org/10.2118/30316-MS.

Hanafy, H. ., Macary, S. M., El Nady, Y. M., Bayomi, A. A., \& El Batanony, M. H. (1997). A New Approach for Predicting the Crude Oil Properties. Society of Petroleum Engineers. https://doi.org/10.2118/37439MS.

Hemmati, M. N., \& Kharrat, R. (2007). A Correlation Approach for Prediction of Crude-Oil PVT Properties. Society of Petroleum Engineers, 1-15. https://doi.org/10.2118/104543-MS.

Izurieta, A. J., Iza, A. C. (2017). The PVT Properties of the Ecuadorian Crude Oils. Society of Petroleum Engineers, 1-29. https://doi.org/10.2118/185483-MS.

Iza, A. (2017). Análisis y Generación de Correlaciones PVT del Petróleo y su Aplicabilidad en el Ecuador. (Tesis de grado) Quito, Ecuador: Escuela Politécnica Nacional.

Jiménez, T. y Zurita, K (2019). Generación de Correlaciones de Presión, Volumen e Temperatura para el Petróleo de los Activos Andes, Auca y Repsol. (Tesis de grado) Quito, Ecuador: Escuela Politécnica Nacional.

Jr, W. D. M., \& Valko, P. P. (2002). Reservoir oil bubblepoint pressures revisited; solution gas - oil ratios and surface gas specific gravities. J Pet Sci \& Eng, 37(2003), 153169. https://doi.org/10.1016/S0920-4105(02)00319-4.

Kartoatmodjo, T., \& Schmidt, Z. (1991). New Correlations for Crude Oil Physical Properties. Society of Petroleum Engineers. Obtenido de la base de datos OnePetro.

Khan, S. A., Al-Marhoun, M. A., Duffuaa, S. O., \& AbuKhamsin, S. A. (1987). Viscosity Correlations for Saudi Arabian Crude Oils. Society of Petroleum 
Engineers, 251-258. https://doi.org/10.2118/15720MS.

McCain Jr., W. D. (2002). Analysis of Black Oil PVT Reports Revisited. Society of Petroleum Engineers. https://doi.org/10.2118/77386-MS.

McCain, W., Spivey, J., \& Lenn, C. (2011). Petroleum Reservoir Fluid Property Correlations, 35-133. Tusla: PennWell.

Murillo, J. y Yépez, C (2013). Recopilación de los Análisis P.V.T. y Análisis Convencional de Núcleos para la Generación y Estandarización de Correlaciones para los Campos del Área Auca. (Tesis de grado) Quito, Ecuador: Escuela Politécnica Nacional.

Muzammil, R \& Abdulazeez, A. (2015). PVT correlations for Pakistani crude oils using artificial neural network, 217-233. Springer. Obtneido de: https://link.springer.com/content/pdf/10.1007/s13202016-0232-z.pdf

Petrosky, G. E., \& Farshad, F. F. (1995). Viscosity Correlations for Gulf of Mexico Crude Oils. Society of Petroleum Engineers. https://doi.org/10.2118/29468MS.

Petrosky Jr., G. E., \& Farshad, F. (1993). Pressure-VolumeTemperature Correlations for Gulf of Mexico Crude Oils. Society of Petroleum Engineers, 1(5), 395-406. https://doi.org/10.2118/26644-MS.

Standing, M. B. (1947). A Pressure-Volume-Temperature Correlation For Mixtures Of California Oils And Gases. Drilling and Production Practice, API, 275287. Obtenido de la base de datos OnePetro.

Velarde, J., Blasingame, T. . A., \& McCain Jr, W. D. (1997). Correlation of Black Oil Properties at Pressures Below Bubble Point Pressure-A New Approach. Petroleum Society, 38(13), 10. https://doi.org/10.2118/97-93.

\section{BIOGRAFÍAS}

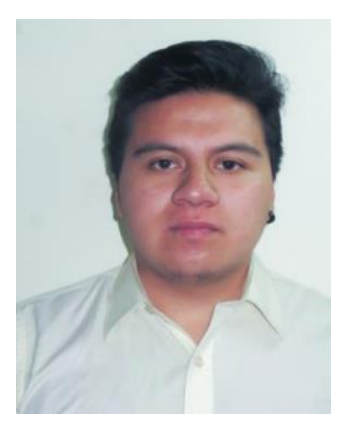

Jimmy Darío Arteaga Tuz. Estudiante de la Facultad de Geología y Petróleos, desde los primeros semestres mostro una gran habilidad en la programación y desarrolló una visión de cómo la programación combinado con la Ingeniería en Petróleos puede ayudar al País desarrollando software Nacional. Actualmente se encuentra en desarrollo de cuatro algoritmos de Ingeniería en Petróleos, incluido el de la presente investigación, todo esto para la implementación de software Nacional en la industria. Sus intereses actuales son: creación y posicionamiento de empresas en cualquier ámbito, finanzas personales y empresariales, administración de talento humano e inversiones en la bolsa de valores. Identificador digital ORCID: https://orcid.org/0000-0002-8116-1409.

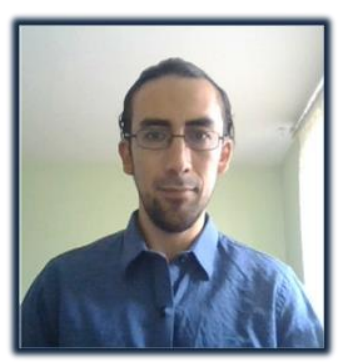

Franklin Vinicio Gómez Soto. Ingeniero en Petróleos de la Escuela Politécnica Nacional (2010), Asistente de Cátedra en el Departamento de Petróleos de la EPN (2011-2012). Hizo la maestría en Termodinámica de Fluidos en la Universidad de Valladolid, España (UVA, 2012). Su proyecto de fin de master fue publicado en "The Journal of Supercritical Fluids", ELSEVIER. Trabajó como Coordinador Petrolero en Triboil Gas CIA. LTDA durante el año 2013 y 2014. Se reincorporó a la Escuela Politécnica Nacional como docente titular Auxiliar desde octubre del año 2014. Es jefe del Laboratorio de Fluidos de Perforación y Petrofísica de la EPN. Se interesa en Fracturamiento Hidráulico y Reacondicionamiento de pozos (reservorios de petróleo y gas). Identificador Digital ORCID: https://orcid.org/0000-0003-4367-4972.

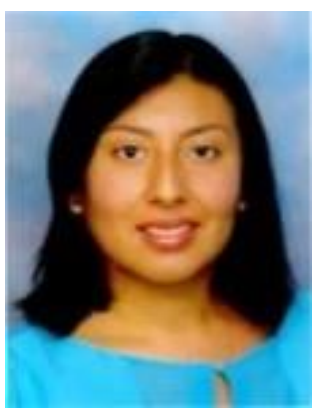

Anabel Cristina Iza Toapanta. Obtuvo el Diploma de Bachillerato en Ciencias especialidad FísicoMatemáticas (Unidad Educativa Liceo Naval, 2010). Se graduó de Ingeniera en Petróleos de la Escuela Politécnica Nacional (2017). Realizó prácticas pre-profesionales en diversas empresas como Sólidos y Lodos Nacionales S.A., Petroamazonas y OCP en el año 2016. Colaboró en un proyecto interno para el Departamento de Petróleos de la EPN (2018-2019). Coautora de un artículo académico publicado en la Sociedad de Ingenieros Petroleros (SPE). Participó como expositor en el evento Sinergia 2019 de la EPN y en el Next Wave Paper Contest 2019 de la SPE Sección Ecuador. Identificador digital ORCID: https://orcid.org/0000-00023097-1313.

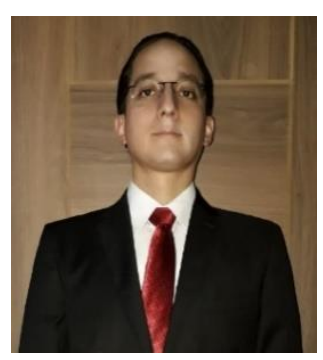

Álvaro Javier Izurieta Ballesteros. Ingeniero en Petróleos, Escuela Politécnica Nacional (2010), Especialista Industria Petroquímica, Universidad Nacional General San Martin (2017) se desempeña actualmente como Asesor Técnico para Halliburton. Sus intereses son la optimización de producción mediante fracturamiento hidráulico, estimulación acida, ingeniería de producción de petróleo e ingeniería de producción. Es autor y coautor de varios estudios técnicos para la Sociedad de Ingenieros Petroleros (SPE) y Congreso Mexicano del Petróleo (CMP). Identificador digital ORCID: https://orcid.org/0000-0002-5615-5621. 


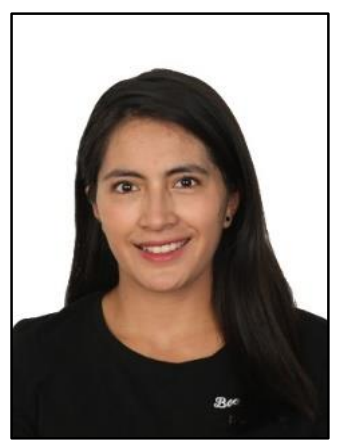

Tamia Fernanda Jiménez Escobar. Obtuvo el Diploma de Bachillerato en Ciencias General (Colegio Técnico Experimental de Aviación Civil "COTAC", 2011). A partir de ese momento supo que quería estudiar una ingeniería y graduarse con excelencia lo cual lo lograría en la Escuela Politécnica Nacional al graduarse de Ingeniera en Petróleos (2019). Durante su carrera trabajo en varias empresas de Petróleo como pasante en las cuales están Halliburton y Schlumberger en el año 2017 y Petrobell en 2018. Una vez titulado se fue a perfeccionar el idioma inglés al Reino Unido. Está interesada en trabajar en una empresa internacional que se ocupe en Operaciones en el país. Identificador digital ORCID: https://orcid.org/0000-00021248-6402.

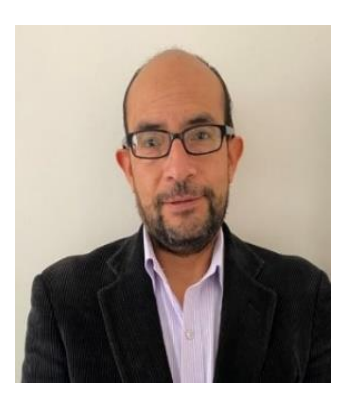

Raúl Armando Valencia Tapia. Ingeniero en Petróleos y Magister en Ingeniería Industrial, ambos de la Escuela Politécnica Nacional. Se desempeña como profesor Titular a tiempo completo en la carrera de Ingeniería en Petróleos desde el año 1998; además, fue profesor en la Facultad de Ciencias de la Ingeniería en la Universidad Tecnológica equinoccial. Desde hace siete años hasta la actualidad se desempeña como Jefe del Departamento de Petróleo. Sus intereses en investigación se centran el Área de Petróleos, específicamente en la Línea de Yacimientos de Hidrocarburos y Recuperación Mejorada. Identificador digital ORCID: https://orcid.org/0000-0001-8341-8864.

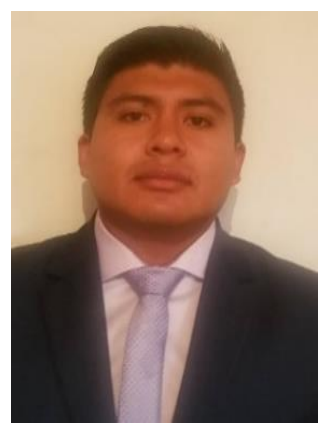

Kevin Fabricio Zurita Pedraza. Bachiller en la especialidad FísicoMatemático (Unidad Educativa Municipal Sucre, 2011), participó en las Olimpiadas Matemáticas realizadas en la EPN, 2010. Se gradúo de Ingeniero en Petróleos en la Escuela Politécnica Nacional en 2019, fue ayudante del Laboratorio de Fluidos de Perforación de la Facultad de Geología y Petróleos. Actualmente Docente de Matemáticas del Colegio Virtual José Moncada Sánchez. Esta interesando en libros de aportes de la ciencia a la humanidad y deportes como natación. Identificador digital ORCID: https://orcid.org/0000-0003-1446-2241. 
Revista Politécnica, Agosto - Octubre 2020, Vol. 46, No. 1 